\title{
Improved Single Haze Removal using Weighted Filter and Gaussian-Laplacian
}

\author{
Ajeeta Singh Bhadoria ${ }^{1}$, Vandana Vikas Thakre ${ }^{2}$ \\ ${ }^{I}$ M. Tech Scholar, Department of Electronics \&Comm. Engineering, MITS, Gwalior Ajitabhadoria12@gmail.com, \\ ${ }^{2}$ Associate professor, Department of Electronics \& Comm. Engineering, MITS, Gwalior vandana@mitsgwalior.in \\ *Correspondence: Ajeeta singh bhadoria, Ajitabhadoria12@gmail.com
}

\begin{abstract}
Generally computer applications use digital images. Digital image plays a vital role in the analysis and explanation of data, which is in the digital form. Images and videos of outside scenes are generally affected by the bad weather environment such as haze, fog, mist etc. It will result in bad visibility of the scene caused by the lack of quality. This paper exhibits a study about various image defogging techniques to eject the haze from the fog images caught in true world to recuperate a fast and enhanced nature of fog free images. In this paper, we propose a simple but effective the weighted median (WM) filter was first presented as an overview of the standard median filter, where a nonnegative integer weight is assigned to each position in the filter window image .Gaussian and laplacian pyramids are applying Gaussian and laplacian filter in an image in cascade order with different kernel sizes of gaussian and laplacian filter .The dark channel prior is a type of statistics of the haze-free outdoor images. It is based on a key observation - most local patches in haze-free outdoor images contain some pixels which have very low intensities in at least one-color channel. Using this prior with the haze imaging model, we can directly estimate the thickness of the haze and recover a high-quality haze-free image. Results on a variety of outdoor haze images demonstrate the power of the proposed prior. Moreover, a high-quality depth map can also be obtained as a by-product of haze removal and Calculate the PSNR and MSE of three sample images.
\end{abstract}

Keywords: Image Defog, DCP, IDCP, Weighted filter, Gaussian-laplacian filter.

\section{Introduction}

Image processing (IP) systems increment the estimation of image |from the defiled picture. Outside scene pictures captured within the weather condition are typically attributable to the entity of the haze, fog, mist, or different media attributable to weather condition atmosphere fog, haze, rain and snow is that the major reason of image degradation. During fog the light gets detached before reaching the camera, once we take a picture employing a camera thanks to a touch impurity within the atmosphere.

\section{ARTICLE INFORMATION}

Author(s): Ajeeta Singh Bhadoria, Vandana Vikas Thakre;

Received: June 30, 2020; Revision: July 17, 2020; Published: July 20,

2020;

e-ISSN: 2347-470X; Paper Id:IJEER-080201;

Citation: https://doi.org/10.37391/IJEER.080201

\begin{tabular}{|l|}
\hline CrOSS \\
\hline CROSSREF \\
THECITATION IINING BACKBONE \\
\hline
\end{tabular}

Webpage-link: http://ijeer.forexjournal.co.in/archive/volume-8/ijeer-080201.html

Image Fusion may be a mechanism to boost the standard of knowledge from a collection of pictures. By the method of image fusion the great info from every of the given pictures is fused along to make a resultant image whose quality is superior to any of the input pictures. This can be achieved by applying a sequence of operations on the photographs that will create the great information in every image distinguished. The resultant image is created by combining such magnified information from the input pictures into one image. Image Fusion finds it application in wide variety of areas. It is used for medical nosology and treatment. A patient's pictures in several information formats are often united. These forms will embody resonance image (MRI), X-raying (CT), as an example, CT pictures are used a lot of usually to determine variations in tissue density whereas imaging pictures are usually wont to diagnose brain tumors. Image fusion is additionally utilized in the sphere of sensing wherever variable pictures like thermal pictures, IR Images, UV Images [2].

Image enhancement technique enhances the haze image however it result in loss of data in image. Image Restoration (IR) is concerned with the physical procedure of imaging in fog. Image can really be set within the wake of observance debasement of haze and cloudiness. Finally, the corruption procedure is employed to create the fog free image. In principal systems, connected image visibility degradation are often tough that run underneath a broad range of atmospheric condition, together with out of doors visual perception systems, remote sensing systems, sensible transportation systems like recording of moving vehicle data and traffic observation systems. By the presence of floating tiny particles within the atmosphere, haze is created that is called aerosols. This can be able to grasp and unfold the beams of sunshine right away within the field of computer vision. Image dehazing has end up to be a big analysis drawback. The getting ready of image that is foggy is fairly difficult. Sadly the nature of mist is eccentric i.e., the murkiness impacts changes over the scene. As it were, displaying with varied photos mist or murkiness demonstrate is not typically acceptable. Old researchers uses the normal 
techniques from one image, science is to get rid of the haze. That's the reason; the dehazing impact is strained in lightweight of the actual fact that single hazy image will nearly not offer abundant knowledge. Afterward, with completely different photos analysts endeavor to enhance the dehazing execution. Projected haze removal techniques with multiple pictures of a similar scene underneath varied atmospheric condition. The work, is supported on two observations. Within the initial place, cloudiness free photos have additional complexness than photos corrupted by terrible climate; second, the forms of air lightweight, that depends upon the separation between the things to the watcher. As of late, varied examinations have organized on single image way to deal with reestablishing the perceivability of a murky image. These procedures rely on either solid suspicion or hearty priors, by that fog thickness is probably going by utilizing simply a solitary image. A murkiness free image has clear stands out investigate from a murky image. Utilizing of assessment of sort of scene differentiate this system reestablishes the deceivability of an image by boosting its neighborhood differentiate [1].

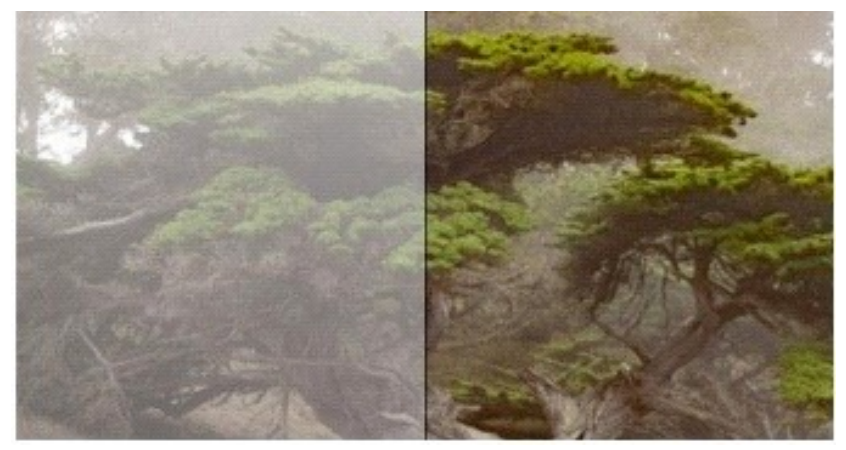

Figure 1. Original Image and Fog Removal Image

\section{Various Defogging Techniques}

\subsection{Text Dark Channel prior}

This technique is actually helpful for defogging of single image .The pixels of the fog free image is measured. Imagine that in any of the colour channel, extraordinarily least intensity of few pixels. These pixels are outlined as dark pixels. These dark pixels are costumed to calculate the transmission map. Transmission map is to eliminate some block result. Single image is utilized for foggy image restoration (IR) so Transmission map is predicted exactly essentially, the minimum intensity in such a small amount ought to have terribly lowest worth. Dark channel is intended by, Dark(x) = $\min \}, Y \in \Omega(x) c \in$ wherever $J^{\prime}$ could be a color channel of $J$ and $\mathrm{Q}(\mathrm{x})$ could be a native.

\subsection{Improved dark channel prior (IDCP):}

IDCP was proposed by Yan WangBo Wu within the year 2010. Same idea as employed by DCP however at same time provides improvement for estimation of air-light by increasing the patch size to thirty one $\times$ thirty one. Time complication of algorithmic program reduces since it avoids creating use of sentimental matting technique.

\subsection{Weighted filter}

In weighted filter, we tend to give a lot of weight to the central value, so the contribution of center value becomes more than rest of the values. Due to weighted average filtering, we will manage the blurring of image. These filters are applied by replacement of every pixel intensity by a weighted average of its neighboring pixels. The weights that are applied to the neighboring component intensities are contained during a matrix referred to as the convolution matrix. This filter emphasizes contours within the image [4].

\subsection{Gaussian-laplacian pyramid}

Gaussian and laplacian pyramids applying Gaussian and laplacian filter in a picture in ca. the weather of a Gaussian Pyramids area unit ironed copies of the image at totally different scales [5].

\section{Literature Survey}

Higang Lingietial. [2017] during this paper, our will is to build up a completely unique image defogging calculation by specifically anticipating the haze thickness of recovered pictures as opposed receiving earlier assumptions or necessities. Thus on accomplishing this objective, two specific advances are conferred. To begin with, we tend to embrace three fog vital time asurable highlights got from foggy pictures, and more over build up a basic fog density evaluator (SFDE) by creating a linear merge of those fog-relevant features. This proposed evaluator will proficiently see the fog density of a solitary image while not respect to a relating fogfree image associated contains a low machine load compared with an existing methodology. Second, a material science based mostly numerical association between the transmissions and therefore the mist thickness score of the recuperated image is formed by strategies for SFDE; therefore image defogging will act sort of a minimization issue on the mist thickness score of the recouped picture [6].

Changli Liiietial. [2017] this text principally focus of the image restoration. 1st of all, it studies the side fogging algorithmic program based mostly on DCP and create some improvement based mostly on this theory. in resolution the defects of estimation of filled with atmosphere lightweight and very long time running of the is algorithmic program, the advance estimation of environmental condition lightweight and coefficient are introduce during this paper to boost the coefficient of estimation by introducing a gain constant rather soft matting algorithmic program for very long time. At the similar time four binary tree subdivision strategies is within the habit of estimate the region lightweight, that is ready to shorten the operation time, avoid the halo development and find associate improved defogging out return [7].

Md. Imtiyaz Anwarietial. [2017] during this paper, it's planned for visibility ehancement through fog removal employing a single grey or a color image, by a good post-processing technique. This algorithmic program relies on DCP conception 
followed by a post-processing methodology based mostly visibility enhancement technique for foggy pictures. Subjective and quantitative investigation is connected for assessment of defogged pictures procured from the planned methodology and is in like manner differentiate and different actually understood techniques to found its potency. The planned algorithmic program preserves sharp details and maintains the colour worth of the defogged image [8].

Jaiveer Singh Sikarwarietial. [2016] this has been appeared in existing exploration that the massive fraction of the current systems has varied problems. To beat the constraints of the former work; a completely unique system has been existing during this paper. During this analysis, changed dark channel previous (MDCP) and Gauss-Laplacian filtering (GLP) with transmission map. The planned work is existent in MATLAB utilizing image dispensation tool cabinet. The comparison among normalization technique and therefore the planned algorithmic program is measured differing kinds of performance, namely, the peak to signal noise ratio (PSNR), entropy and execution time. The experimental results have composed of dissimilar kinds of fog image and it's shown higher results as compared to previous strategies [9].

XinNing, etial. [2015] this paper projected an image fog elimination method. This strategy in view of the properties of retinal neurons concentric open field whose capacity is three Gaussians. Firstly, by utilizing contrast constrained worldwide histogram evening out we enhance unique mist picture. Secondly, to ire-establish the images details and penetration information a local image improvement method is passed out. According to experiment, the results of this paper's method were compare with the results of DCP based method and the results of CLAHE. The new results it was able to avoid enhancing too light and to avoid adding noising it has proved that the method of CLAHE. The method of this paper worked well in images fog removing and enhanced fog images' visibility. [10].

\section{Propose Work}

In this effort we have proposed a new view that will effectively solve insufficient fog breadth evaluation and color cast problem. By doing so, we are going to make hazed image to haze-free and with accurate calculation of the atmospheric light which is hard to calculate due to the presence of the sky in the image and thus we using weighted filter and Gaussianlaplacian pyramid.

\subsection{Propose algorithm}

STEP 1: First run out program and get the output STEP 2: F irst Browse our Original Haze-Image.

STEP 3: Applying dark channel prior algorithm.

STEP 4: Estimate the Atmospheric light.

STEP 5: Scene Radiance by Dark Channel.

STEP 6: Getting Image by Improving the Radiance.

STEP 7: Getting images by Transient Image.

STEP 8: Image improvement by proposed technique.

\subsection{MSE (Mean Square Error)}

If comparison of two images is made based on their respective qualities then a metrics called MSE is used. The MSE between two images $\mathrm{g}(\mathrm{x}, \mathrm{y})$ and $\mathrm{f}(\mathrm{x}, \mathrm{y})$ is

$$
s_{M S E}=1 / M N \sum_{n=1}^{M} \sum_{m=1}^{N}[f(n, m)-g(n, m)]^{2}
$$

Where $\mathrm{M}$ represent the number of rows and $\mathrm{N}$ represent the number of columns respectively.

\subsection{PSNR (Peak signal-to-noise ratio)}

One of the issues with the MSE, the PSNR keeps away from (MSE depends intensely on the picture force scaling) by scaling MSE as per the picture extend.

$$
\text { PSNR }=-10 \log _{10} \frac{e_{\text {MSE }}}{Q^{2}}
$$

Where $\mathrm{Q}$ is the maximum pixel value.

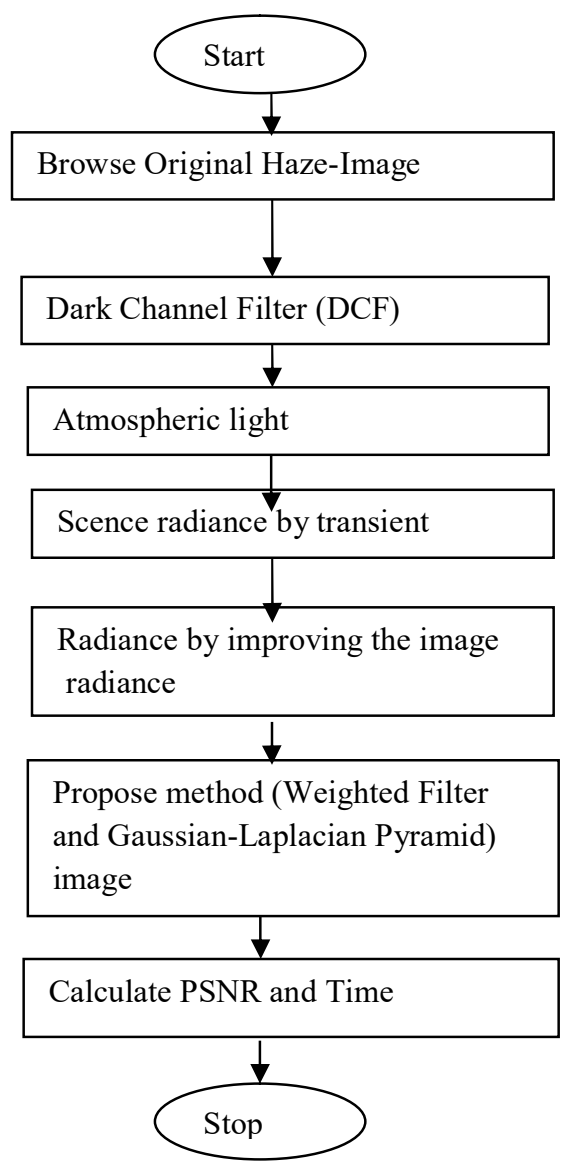

Figure 2. Flowchart of propose work

In flow chart First we are going to browse the image and then we are going to apply dark channel Filter The dark channel is 
a type of outdoor haze-free images. This easy technique depends on the dark channel prior which is more powerful than the "brightest pixel" method. We use it to conventionally approximate the atmospheric lights for all images. It depends on an approximation - most local marks in outdoor haze-free images consists some pixels whose strength is less in partially one color channel and then we are going to use scene radiance. Radiance is the flux releases, reflected, transferred or acquired by a particular surface per unit solid angle and projected area. Historically, radiance is called "intensity" and spectral radiance is called "specific intensity". On our proposed technique we apply weighted filter. These filters are used by returning each pixel intensity by a weighted average of its neighboring pixels. The weights that are used to the neighboring pixel intensities were hold in a matrix known as the convolution matrix. We use gaussian-laplacian pyramid.

\section{Result Analysis}

In this work we have merged two algorithms for getting better we propose multi objective differential evolution based streamling for picture defogging utilizing contrast gain and level of saturated pixels. We would like to emphasize that although weighted filter have some similarities with linear filters, weighted filter cannot replace linear filter and vice versa. We have shown through peak signal to noise ratio and mean square root values comparison of hazed and haze free image.

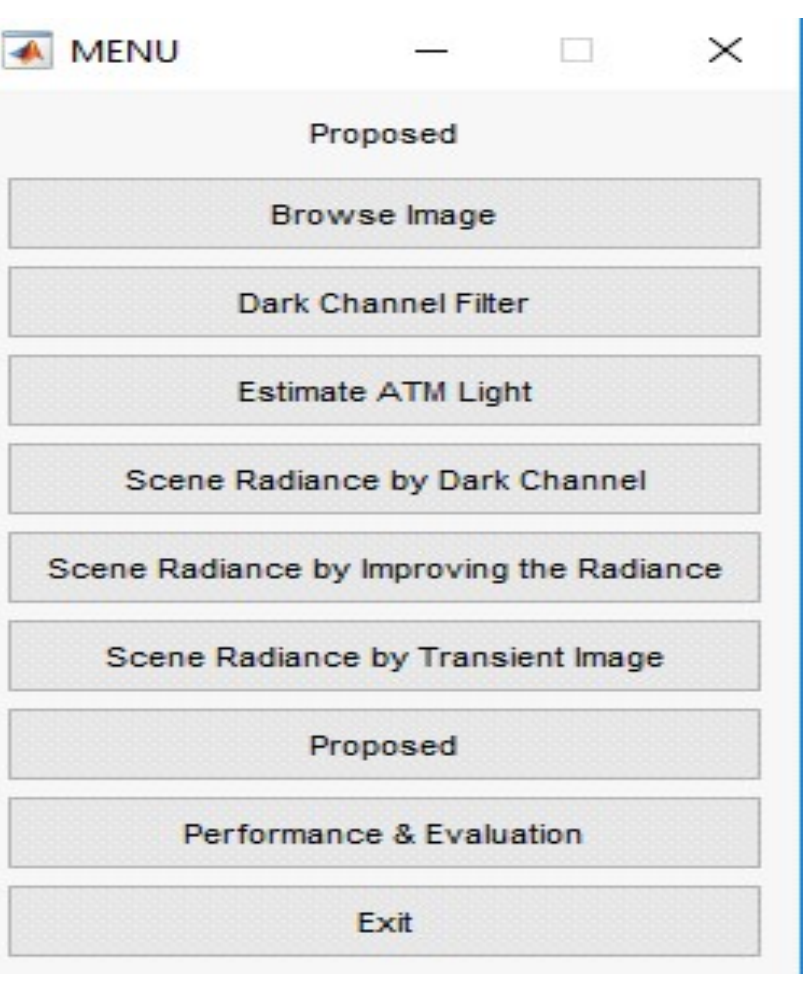

Figure 3. First we run out program and get the output.

In above figure we run the program according to our requirement and getting the desired output by applying multiple techniques.

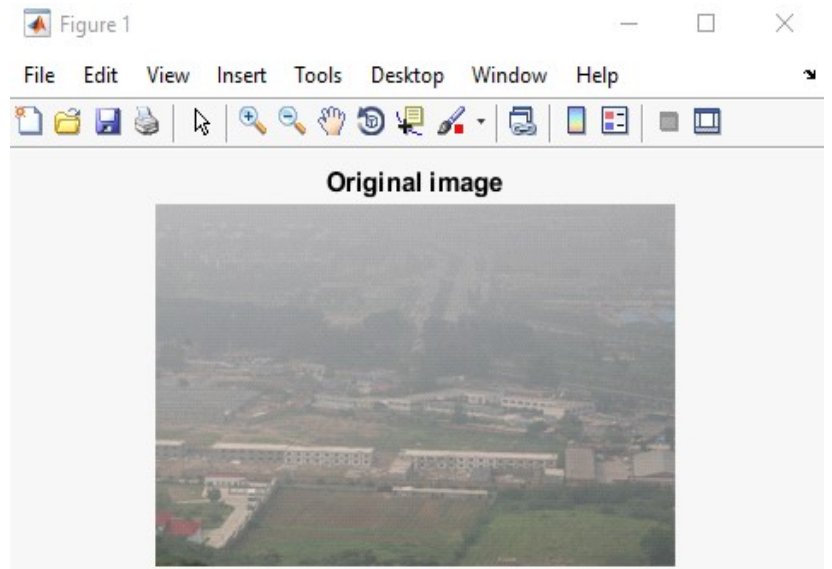

Figure 4. Browseanoriginal haze-image

In the above figure, we browse an original haze image. The turbid medium generally deteriorates images of scenes in the airspace. Haze, fog, and smoke are situations due to atmospheric absorption and dispersion. The scene point is diminished besides the line of sight is called irradiance which gets by the camera.

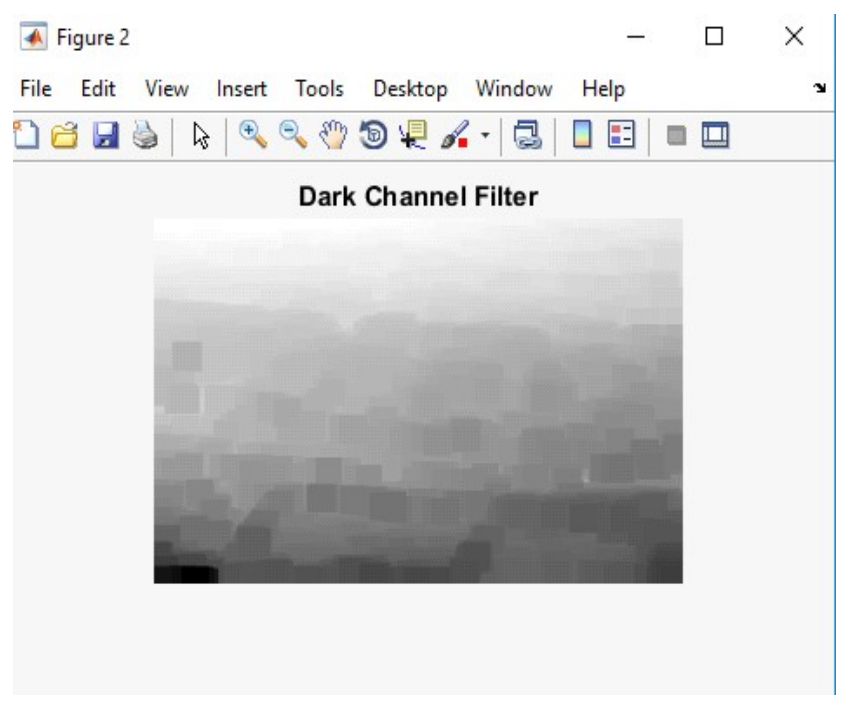

Figure 5. Apply dark channel prior on image.

In above figure, the dark channel prior applied on original image which depends on the figures of haze-free outdoor images. We gets that most of confined part which do not wrap the sky, it is frequent that "dark pixels" have less intensity in no less than one (rgb) channel. In a haze image, the strength of dark pixels is given by the light. Consequently, these dark pixels can supply an accurate evaluation of the haze's transmission. 


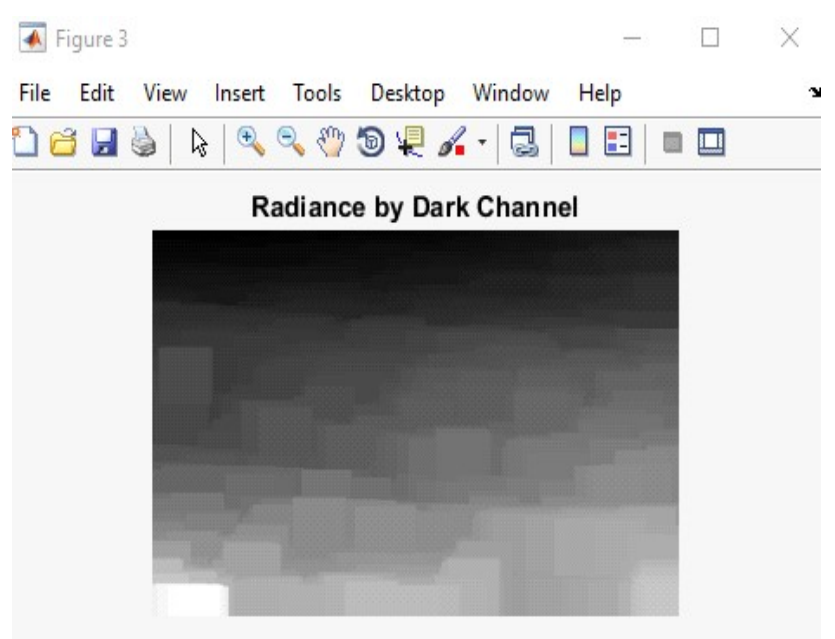

Figure 6. Scene Radiance by Dark Channel.

In above figure we improve image radiance by scene radiance.

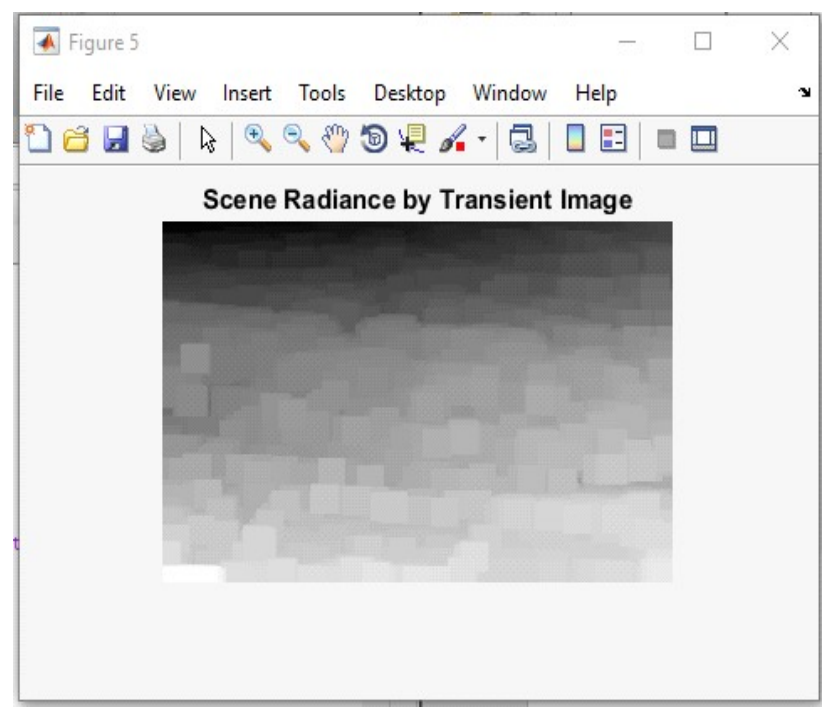

Figure 8. Getting images by Transient Image.

In above figure, we get the image by transient method. A transient image is a series of images which envision the transmission of light during an ultra-short time period. A transient image is the optical impulse result of a scene, which also visualize the emission of light during an ultra-short time interval.

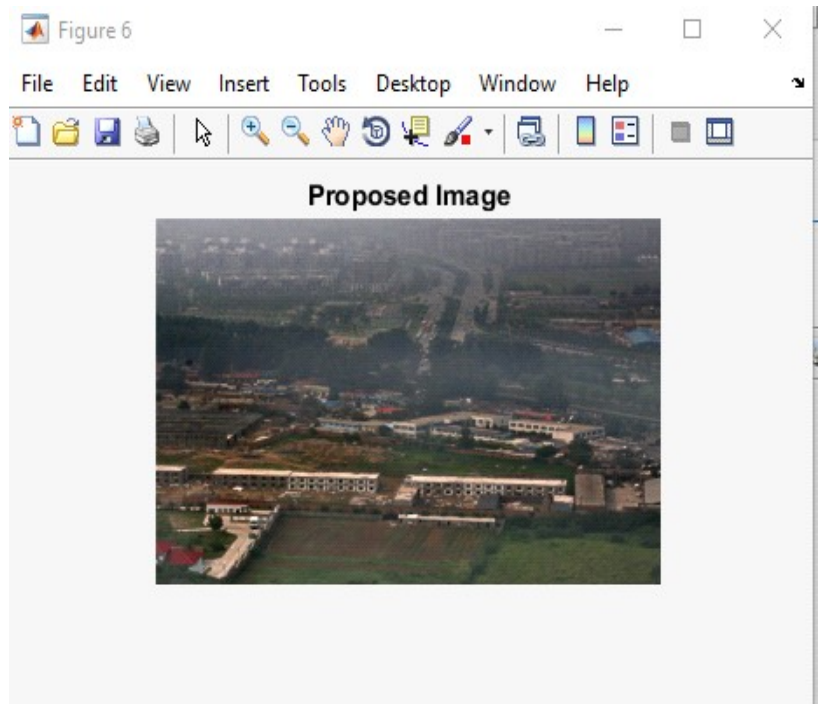

Figure 9. Image improvement by proposed technique.

In above figure, we get the improved image by applying propose techniques. On our proposed technique we apply weighted filter. These filters are applicable by returning each pixel intensity by a weighted average of its neighboring pixels. The weights that are used to the neighboring pixel intensities are stored in a matrix called the convolution matrix. We use Gaussian-laplacian pyramid.

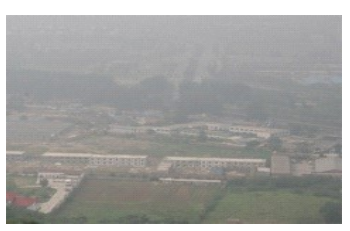

(a) Hazed image

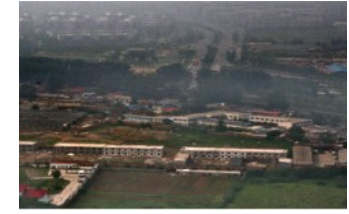

(b) Haze free Image Figure 10. Sample image

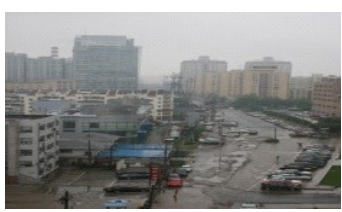

(c) Hazed image

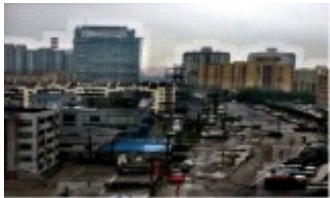

(d) Haze free Image Figure 11. Sample image 2

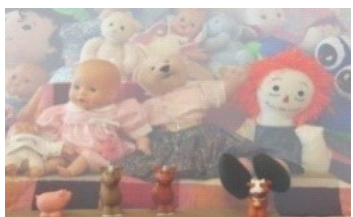

(e) Hazed image

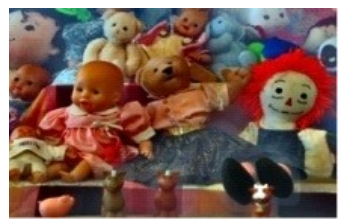

(f) Haze free Image
Figure 12. Sample image 3

In above figures, we have taken three image and applying different techniques with proposed approach, we get the haze 
free image. We have proposed a weighted filter with Gaussian laplacian pyramid, as well as the dark channel prior, for single image haze removal. The dark channel depends on the figures of outdoor haze-free images. Combining the prior with the haze imaging model, haze removal becomes easier and efficient. Since the dark channel prior is a kind of statistics, cannot work for some images. When the objects are inherently alike to the atmospheric light and no shadow is cast on them the dark channel prior is invalid. The dark channel of the scene radiance has bright values near such objects. As a result, our method will underrate the transmission of these objects and overrate the haze layer.

Table 1. Comparisons peak signal to noise ratio of the: Sample Images:

\begin{tabular}{|c|c|c|}
\hline Image & Base & Propose Base \\
\hline Sample Image 1 & 36.8150 & 47.1373 \\
\hline Sample Image 2 & 37.4457 & 47.0876 \\
\hline Sample Image 3 & 36.7209 & 47.0663 \\
\hline
\end{tabular}

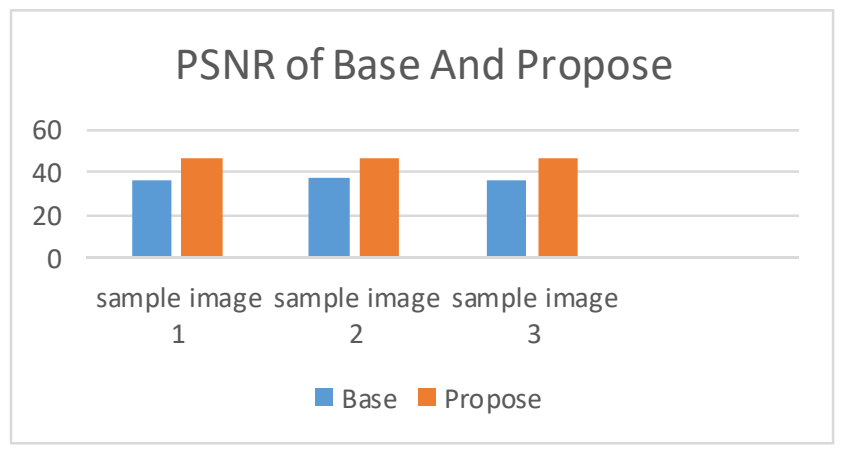

Figure13.Comparisons previous techniques and new techniquefor PSNR

Table 2. Comparisons MSE of the Sample Images

\begin{tabular}{|c|c|c|}
\hline Image & Base & Propose \\
\hline Sample Image 1 & 13.6453 & 1.2815 \\
\hline Sample Image 2 & 11.8009 & 1.2669 \\
\hline Sample Image 3 & 13.9442 & 1.2878 \\
\hline
\end{tabular}

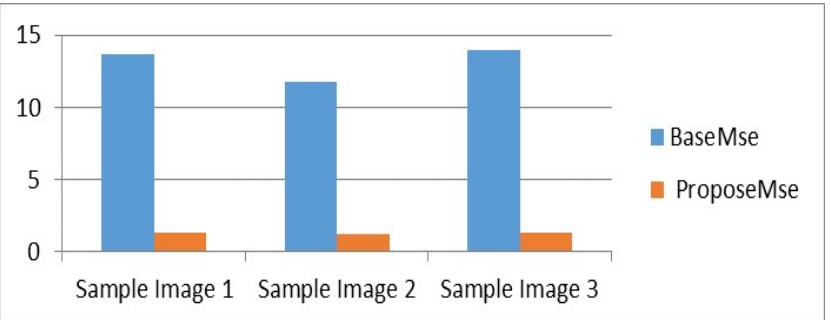

Figure14.Comparisons previous techniques and new technique for MSE
In table 1 and fugure13, we compared the three sample image PSNR values using our techniques with proposed approach and in table 2 and figure 14, we compared the three sample image MSE values using our techniques with proposed approach.

\section{Conclusion}

In this paper, we proposed an easy but powerful filter, weighted filter, for single image haze removal and Gaussian laplacian pyramid. The dark channel prior depends on the figures of the outdoor images. Applying the prior into the haze imaging model, single haze removal becomes more efficient. The dark channel prior is a measure, which will not work for some images. When the objects are alike to the atmospheric light and no shadow is cast on them, the dark channel prior is invalid. Our work also covers the restrictions of most haze removal methods - the haze imaging model may be invalid. More advanced models (using weighted filter and Gaussian laplacian pyramid) were used to narrate complex situation, such as the sun's influence on the sky region, and the bluish hue near the horizon. We determine to approximate haze removal on these models in the future.

\section{REFERENCES}

[1] Mayuriv Badhe, Prof. Prabhakal Ramteke, "A Survey on Haze Removal using Image Visibility Restoration Technique". International Journal of Computer Science and Mobile Computing IJCSMC, Vol. 5, Issue. 2, February 2016.

[2] Tupe S. G., Prof. Raskar V.B., "A SURVEY OF HAZE REMOVAL TECHNIQUES FOR IMAGE PROCESSING”. IJARIIE-ISSN (O)-23954396. Vol-3 Issue-3 2017.

[3] Neha Padole1, Akhil Khare, "Improved Method of Single Image Dehazing based on Multi-Scale Fusion”. International Journal of Computer Science and Information Technologies, Vol.6 (3) 2015, 2945-2949.

[4] Ramandeep1, Rajiv Kamboj, "Review on Different Aspects of Image Fusion for Medical Imaging”. International Journal of Science and Research (IJSR) ISSN (Online): 2319-7064. Volume 3 Issue 5, May 2014.

[5] Poonam1 Dr.V K Banga, Gurjit singh, "A Review on Haze Removal Techniques". International Research Journal of Engineering and Technology (IRJET) e-ISSN: 2395-0056 Volume: 04 Issue: 08Aug-2017 ISSN: 2395-007 [6] Mamta Sharma, "A Review: Image Fusion Techniques and Applications". International Journal of Computer Science and Information Technologies, Vol. 7 (3) 2016, 1082-1085.

[7] Manpreet Kaur Saggu†* and Satbir Singh,"A Review on Various Haze Removal Techniques for Image Processing". International Journal of Current Engineering and Technology E-ISSN 2277 - 4106, P-ISSN 2347 $5161 \odot 2015$.

[8] Saleha Masood1, Muhammad Sharif1, Mussarat Yasmin1 Muhammad Alyas Shahid1,* and Amjad Rehman2, "Image Fusion Methods: A Survey" Journal of Engineering Science and Technology Review 10(6) (2017) 186194.

[9] Amla Thomas1* and M. Azath2, "A Survey on Image and Video Dehazing”. International Journal of Computer Sciences and Engineering Vol.3(1), PP (21-23) Jan 2015, E-ISSN: 2347-2693, 2015, IJCSE.

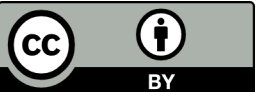

Attribution
(C) 2020 by the authors. Submitted for possible open access publication under the terms and conditions of the Creative Commons $(\mathrm{CC} \quad \mathrm{BY})$ license (http://creativecommons.org/licenses/by/4.0/). 